\title{
Modeling User Navigation
}

\author{
Eelco Herder \\ Department of Computer Science, University of Twente \\ P.O. Box 217, 7500 AE, Enschede, The Netherlands \\ herder@cs.utwente.nl
}

\begin{abstract}
For providing users with navigation aids that best serve their needs, user models for adaptive hypermedia should include user navigation patterns. This paper describes elements needed and how these elements can be gathered.
\end{abstract}

\section{Navigation Strategies and Lostness in Hyperspace}

One of the most common problems in the field of hypermedia is that users tend to become disoriented: they lose one's sense of location and direction in non-linear documents. This problem is aggravated by the fact that users need to maintain track of their tasks, their previous actions and their current context. This additional effort has been given the term cognitive overhead. Both disorientation and cognitive overhead may lead users to get 'lost in hyperspace' [7].

Despite several attempts to infer lostness from users' navigation patterns (e.g. [9]), no clear measure has been found yet. This is mainly due to the fact that users who are exploring a document can be rated as disoriented, even though they may be experiencing no disorientation [1]. As users may display various navigation strategies, varying from goal-directed to explorative [3], it is hard to tell from their actions whether or not they feel lost. The document structure has impact on user navigation as well, as one cannot follow non-existing links. For example, densely linked hyperspaces with many cross-references provide more freedom for exploration than hierarchically structured hyperspaces do [5].

Navigation support that is adapted to user goals, preferences and knowledge, and to the context of use, is seen as a proper way of providing adequate navigation support for varying user needs [2]. Equally important to selecting the right navigation suggestions, is the number of suggestions that should be given to the user and the way they should be presented [5]. However, various types of navigation strategies call for different types of navigation assistance [3]. Therefore, user navigation strategy is an indispensable part of a user model for adaptive hypermedia.

\section{Navigation Support that Matches User Needs}

Hypermedia links may or may not be embedded in the content regions. Links within the text are usually associative links, which interlink semantically related concepts. 
Links that are not embedded in the text - such as menus, indexes, and site maps expose a document's primary structure [6] and therewith function as contextual navigation aids [8]. Both types of links can be adapted to the user needs. Disabling, removing or annotating associative links [2] can help users to find relevant items more easily, but does not provide the context information needed to prevent disorientation or cognitive overload. Therefore, many research projects have focused on adaptive contextual navigation aids. These projects can be categorized as either focused on the user's local or global spatial context or on the user's temporal context.

Strategies found in the former category include personalizing or adding textual or graphical views of relevant parts of the document structure [2]. Site maps, contextual menus, direct guiding and recommendations help users to decide where to go. Most strategies in the latter category concentrate on various visualizations of previously visited pages. Several examples are mentioned in [10]. As 58\% of user navigation involves revisits [10], users highly profit from reminders where they have been.

Each form of navigation support addresses one or more user needs with respect to ease of navigation. Unfortunately, one cannot provide users with all contextual information at once: even if sufficient screen space is available, users will most likely be overwhelmed by the quantity of navigation suggestions. Therefore, the best approach is to provide users with only those associative, spatial or temporal navigation aids that match their navigation strategies and address the problems they are experiencing. These issues can be derived from a user navigation model.

\section{Elements of a User Navigation Model}

A user navigation model should be able to recognize user navigation strategies and to predict future navigation strategies. Moreover, it should be able to predict future user problems. It is unlikely that navigation strategies and problems related to lostness can be captured by a single measure. Otter [7] suggests that we could make use of a 'battery of measures', which correlate well with one another and which have been shown to measure lostness to some degree. Since - as argued in the first section - user navigation is highly dependent on a document's structure, observed navigation patterns need to be compared to this structure. Quantitative measures based on the document structure and user navigation paths have shown to be very useful for this purpose [4].

Interesting features of a document's structure include its size, link density, linearity, distances and clustering. Characteristics of user navigation include path length, the amount of backtracking, revisitation patterns and path linearity. Metrics for these features can be derived from adaptive hypermedia literature as well as from graph theory. For an overview of these metrics we refer to [4] and [5]. It is expected that user navigation strategies will be reflected in these quantitative measures of navigation patterns through a hypermedia document. As users may display various navigation strategies, a user navigation model should contain various patterns as observed in different hypermedia structures and in different user contexts. We hypothesize that deviations from these patterns can be regarded as indicators for disorientation. 


\section{Current and Future Work}

In order to make the user navigation model useful for adaptive hypermedia purposes, the metrics, as mentioned before, need to be gathered in real-time. We are implementing these metrics in Scone, a proxy-based programmable framework that provides methods for access tracking, site crawling and page modification [11].

An experiment is planned to provide us with qualitative and quantitative data on user navigation patterns, usability problems as perceived by the user and success measures. We are interested in relations between site structure and navigation strategies, and relations between navigation patterns and perceived lostness. The results will be used for further developing the user navigation model, and for developing suitable adaptation strategies.

\section{Acknowledgements}

This research is part of the PALS project (Personal Assistant for onLine Services). The project is supported by the Dutch Innovative Research Program IOP-MMI. Our research partners are TNO Human Factors and the University of Utrecht.

\section{References}

1. Ahuja, J.S. \& Webster, J.: Perceived disorientation: an examination of a new measure to assess web design effectiveness. Interacting with Computers 14 (1) (2001) pp. 15-29

2. Brusilovsky, P.: Adaptive Hypermedia. User Modeling and User-Adapted Interaction 11 (2001) pp. 87-110

3. Catledge, L.D. \& Pitkow, J.E.: Characterizing Browsing Strategies in the World-Wide Web. Computer Networks and ISDN Systems 27 (6) (1995) pp. 1065 -1073

4. Herder, E.: Metrics for the Adaptation of Site Structure. Proc. of the German Workshop on Adaptivity and User Modeling in Interactive Systems, ABIS02, Hannover (2002) pp. 22-26

5. Herder, E. \& Van Dijk, B.: From Browsing Behavior to Usability Matters. Workshop on Human Information Processing and Web Navigation, HCI 2003, Crete (to appear)

6. Miles-Board, T., Carr, L. \& Hall, W. Looking for Linking: Associative Links on the Web. Proc. $13^{\text {th }}$ ACM Conference on Hypertext and Hypermedia (HT'02), Maryland USA (2002) pp. 76-77

7. Otter, M. \& Johnson, H.: Lost in hyperspace: metrics and mental models. Interacting with Computers 13 (1) (2001) pp. 1-40

8. Park, J. \& Kim, J.: Contextual Navigation Aids for Two World Wide Web Systems. International Journal of Human-Computer Interaction 12 (2) (2000) pp. 193-217

9. Smith, P.A.: Towards a practical measure of hypertext usability. Interacting with Computers 8 (4) (1996) pp. 365-381

10. Tauscher, L. \& Greenberg, S.: How people revisit web pages: empirical findings and implications for the design of history systems. International Journal of Human-Computer Studies 47 (1997) pp. 97-137.

11. Weinrich, H., Buchmann, V., Lamersdorf, W.: Scone: Ein Framework zur evaluativen Realisierung von Erweiterungen des Webs. Tagungsband Kommunikation in Verteilten Systemen - KiVS (2003) pp. 12 